Instituto Internacional de Investigación y Desarrollo Tecnológico Educativo INDTEC, C.A.

DOI: https://doi.org/10.29394/scientific.issn.2542-2987.2017.2.3.5.99-117

OAI-PMH: http://www.indteca.com/ojs/index.php/Revista Scientific/oai

\title{
Uso de Abonos Orgánicos para el Desarrollo Sustentable de la Escuela Técnica Agronómica Salesiana
}

\author{
Autor: Ángel Asdrúbal Torres Moreno \\ Universidad Pedagógica Experimental Libertador, UPEL \\ angelasdrubal 44@hotmail.com \\ Barinas, Venezuela
}

\section{Resumen}

La Escuela Agronómica Salesiana "San José" es una institución agropecuaria que enseña a sus estudiantes el manejo efectivo de animales y plantas, desafortunadamente en los últimos años ha aumentado el uso de fertilizantes químicos para el manejo de sus cultivos, estos aun cuando aumentan el rendimiento en las cosechas son altamente perjudiciales para con las personas, animales y fundamentalmente el suelo, el presente trabajo plantea básicamente la utilización de abonos orgánicos para lograr así un desarrollo sustentable de la misma, teniendo en cuenta que la escuela es una gran productora de estos residuos naturales. La investigación es de tipo cualitativa y se utiliza el método fenomenológico como vía para acceder a las particularidades del tema de estudio, para ello fue necesario recoger la información a través de 4 unidades de estudio que son profesores de la institución educativa los cuales están directamente implicados con el uso de abonos orgánicos o químicos. En este contexto surge como resultado la necesidad impostergable de maximizar el uso de abonos orgánicos en los suelos de la institución, así como disminuir en lo posible el uso de los químicos.

Palabras clave: abonos orgánicos; sustentabilidad; suelos.

Fecha de Recepción: 20-09-2016

Fecha de Aceptación: 07-10-2016 


\title{
Organic Fertilizer Use for Sustainable Development of Agricultural in Salesian Technical School
}

\begin{abstract}
Agronomic Salesian School "San Jose" is an agricultural institution that teaches students effective management of animals and plants, unfortunately in recent years has increased the use of chemical fertilizers for crop management, these even when performance enhancing the crops are highly damaging to people, animals and essentially the soil, this paper basically raised by the use of organic fertilizers in order to achieve sustainable development of the same, considering that the school is a major producer of organic waste. The research is qualitative type and the phenomenological method is used as a way to access the particularities of the subject of study, for it was necessary to collect the information through 4 units of study that are teachers of the school which are directly involved using organic or chemical fertilizers. In this context the urgent need to maximize the use of organic fertilizers in the soils of the institution and reduce as much as possible the use of chemicals is the result.
\end{abstract}

Keywords: organic fertilizers; sustainability; soils.

Date Received: 20-09-2016

Date Acceptance: 07-10-2016 


\section{Introducción}

La Escuela Técnica Agronómica Salesiana "San José" es una institución agrícola en donde en primer lugar se les enseña a sus estudiantes todo lo necesario en el manejo efectivo de animales, plantas y otros derivados y además produce rubros para la venta al colectivo barines, posee una gran cantidad de terreno y animales. Mediante muchas observaciones directas se pudo evidenciar que los suelos son la base fundamental de su sustento, sin embargo, están en menoscabo de sus cualidades físicas, químicas y biológicas naturales a causa de la alta utilización de abonos químicos en algunas áreas productivas de la institución, como lo son fruticultura y horticultura.

En consideración con lo anteriormente descrito la presente investigación fenomenológica consiste, fundamentalmente en concienciar en el uso de abonos orgánicos para lograr el desarrollo sustentable de la Escuela con la posibilidad de que no solamente se logre la sensibilización de la misma en este aspecto, sino también lograr que los estudiantes que ahí se preparan se adiestren en el tema y sean multiplicadores activos una vez egresados del plantel.

Ahora bien, innumerables veces se han señalado con claridad la cantidad de perjuicios que ha traído al ambiente la utilización de los abonos químicos o sintéticos como coadyuvantes en el manejo agrícola de los cultivos, de manera que Gonzáles (2011), en su artículo Contaminación por fertilizantes plantea:

La contaminación por fertilizantes se produce cuando éstos se utilizan en mayor cantidad de la que pueden absorber los cultivos, o cuando se eliminan por acción del agua o del viento de la superficie del suelo antes de que puedan ser absorbidos (pág. 1). 
En este sentido, como bien plantea el autor en lo anteriormente descrito, ocurre la contaminación cuando estos se suministran en mayor cantidad de las necesarias, pero también cuando son eliminados o lavados por razones no controladas, claro que se ha demostrado además que aumentan el rendimiento de las cosechas y por ende las ganancias de los productores, es por esto que ocurre la utilización los mismos. La ventaja que trae la utilización de los agroquímicos, que se puede decir que es única es el rendimiento de las cosechas, cualquier individuo egoísta podría preguntarse ¿Y qué más se desea?

En contraposición se tiene el abono orgánico a los que Durán (2015), alude lo siguiente:

En todo suelo o huerto el abono orgánico es una herramienta imprescindible para poder aportar nutrientes a la tierra para que ésta sea lo suficientemente fértil, y aumentar la actividad de los microorganismos del suelo para que las plantas crezcan y se desarrollen correctamente. El aporte de materia orgánica forma parte de las técnicas que se incluyen en la agroecología. (pág. 1).

En este contexto puede evidenciarse la importancia que tienen los abonos orgánicos para con los suelos y por supuesto, al lograrse la concienciación en el uso los mismos se estarían dando pasos agigantados para lograr un verdadero desarrollo sustentable en el plantel agrícola y ahí se tiene que Brundtland (1987), plantea que el desarrollo sustentable "es aquel que satisface las necesidades de la generación presente sin comprometer la capacidad de las generaciones futuras para satisfacer sus propias necesidades" (pág. 59).

De acuerdo con esa perspectiva, se plantea la siguiente interrogante: ¿Cuál es la importancia del suministro de abonos orgánicos a los suelos de la institución para frenar el deterioro progresivo de los mismos producto del excesivo uso de los químicos? 
De igual forma, se perfila el siguiente propósito: Describir los beneficios del uso de abonos orgánicos para lograr el desarrollo sustentable de la Escuela Técnica Robinsoniana Agronómica Salesiana.

\section{Teoría y conceptos}

\subsection{Abono orgánico}

Los abonos orgánicos son todos aquellos residuos de origen animal y vegetal de los que las plantas pueden obtener importantes cantidades de nutrimentos. El suelo, con el suministro de estos abonos se ve enriquecido no solo con nutrimentos sino que también se mejoran sus cualidades físicas, químicas y biológicas, dicho esto Torres y otros (2002) definen abono orgánico como "fertilizantes de origen natural y de los que depende la agricultura orgánica" (pág. 34) siendo esta última llamada también agricultura ecológica y es aquella que se encarga del manejo de cultivos y suelos sin ningún tipo de agroquímico o pesticida dando énfasis así a la sustentabilidad, por lo que indiscutiblemente la agricultura orgánica y sustentable son inherentes a los abonos orgánicos y viceversa.

Siendo así que los abonos orgánicos son la base de la agricultura orgánica en sí, y por su puesto al usarlos se dejaría de depender en gran medida de los agroquímicos que tanto daño causa al ambiente y por su puesto al hombre.

\subsection{Abonos químicos inherentes a la contaminación}

Es de recordar que el propósito fehaciente de la investigación en cuestión es lograr la aplicación parcial o intensiva de abonos orgánicos para lograr así un desarrollo verdaderamente sustentable, para ello es necesario conocer la asociación directa que tiene la contaminación con los abonos químicos o minerales, con esto no se quiere decir que los mismos son los únicos agentes causales de la contaminación, pero si evidentemente son un 
agravante sobresaliente, en tal sentido se tiene que Gonzáles (2011), también

en su artículo Contaminación por fertilizantes: un serio problema ambiental, manifiesta que:

Los excesos de nitrógeno y fosfatos pueden infiltrarse en las aguas subterráneas o ser arrastrados a cursos de agua. Esta sobrecarga de nutrientes provoca la eutrofización de lagos, embalses y estanques y da lugar a una explosión de algas que suprimen otras plantas y animales acuáticos. Los métodos agrícolas, forestales y pesqueros y su alcance son las principales causas de la pérdida de biodiversidad del mundo. Los costos externos globales de los tres sectores pueden ser considerables. (pág. 1)

Así se tiene que cuando se aplican fertilizantes químicos en altas cantidades, al contrario de ayudar se convierten en perjuicio para la naturaleza, pues al ser lavados o lixiviados van a parar a otros lugares donde pueden ser abonos indirectos también de otros organismos acuáticos no deseados como las algas. Es necesario recalcar que los fertilizantes químicos no solo influyen negativamente en los suelos y el agua, sino que también tienen grandes efectos que van en detrimento de la salud humana, factor que es lo que llama la atención, a tal efecto el mismo autor plantea que:

El problema de los nitratos radica en que pueden ser reducidos a nitritos en el interior del organismo humano, especialmente en los niños de menos de tres meses de edad y en adultos con ciertos problemas. Los nitritos producen la transformación de la hemoglobina a metahemoglobina. La hemoglobina se encarga del transporte del oxígeno a través de los vasos sanguíneos y capilares, pero la metahemoglobina no es capaz de captar y ceder oxígeno de forma funcional. La cantidad normal de metahemoglobina no excede el $2 \%$. Entre el 5 y el $10 \%$ se manifiestan los primeros signos de cianosis. Entre el 10 y el $20 \%$ se aprecia una insuficiencia de oxigenación muscular y por encima del $50 \%$ puede llegar a ser mortal (pág. 1). 
Entiéndase por cianosis una coloración azul de la piel y de las mucosas producto de una deficiente oxigenación, y obsérvese con detenimiento y preocupación cuando la insuficiencia de oxigenación supera el $50 \%$ puede llegar a ser mortal en las personas.

\subsection{Beneficios en General de los Abonos Orgánicos.}

No se bebe seguir hablando de abonos orgánicos sin mencionar los grandes beneficios que traen los mismos al ambiente y personas en general a lo cual Martínez C. y otros (1999):

Aunque los abonos orgánicos contienen una concentración baja de nutrimentos en comparación a los fertilizantes químicos, la disponibilidad de estos es más constante durante el desarrollo de los cultivos por la mineralización gradual y natural a la que están sometidos los materiales orgánicos (pág. 13).

De acuerdo con el autor, la liberación de los nutrimentos a los suelos y posteriormente a los cultivos por parte de los abonos orgánicos es más constante que la de los fertilizantes químicos, lo que beneficia indiscutiblemente a los cultivos durante su desarrollo y por su puesto a los suelos debido a que hay una presencia continua de nutrimentos en sus características intrínsecas.

Es importante e imprescindible agregar que más adelante, el mismo autor, en la misma página, aclara: "el abono orgánico está considerado como un abono universal por el hecho de que aporta casi todos los nutrimentos que las plantas requieren para su desarrollo" (pág. 13), en torno a esto, se hace notar una posición más radical y explícita al respecto de la calidad de los abonos orgánicos, catalogándolos como universales en el desarrollo de los cultivos, reforzando así la propuesta de la esta investigación, la cual plantea que si puede existir una reducción parcial o casi total de los fertilizantes químicos y podría usarse con toda confianza los abonos orgánicos. 
Ahora bien, de acuerdo con Trinidad (2011) "los abonos orgánicos influyen favorablemente sobre las características físicas del suelo, estas características son: estructura, porosidad, aireación, capacidad de retención de agua, infiltración, conductividad hidráulica y estabilidad de agregados" (pág. 2), en tal sentido, las características físicas de los suelos están muy presentes al momento de la labranza de la tierra o preparación del terreno para la siembra, por ejemplo la estructura, la cual según el autor, también es modificada positivamente por los abonos orgánicos lo que facilita en gran medida la mecanización de los mismos.

En el mismo orden de ideas, los abonos orgánicos, también alteran favorablemente las características químicas de los suelos por lo que también Trinidad (2011) dice "las características químicas del suelo que cambian por la aplicación de abonos orgánicos son obviamente el contenido de materia orgánica; derivado de esto aumenta el porcentaje de nitrógeno total, la capacidad de intercambio de cationes, el pH y la concentración de sales" (pág. 3), por lo tanto, obsérvese que la parte química de los suelos también se beneficia de la aplicación de los abonos orgánicos, por ejemplo el $\mathrm{pH}$, el cual de forma imperante debe ser neutro en los suelos al momento del establecimiento de los cultivos.

Así mismo, las características biológicas también cambian en forma favorable al aplicársele materiales orgánicos a los suelos y Trinidad (2011) continua "se debe a que los estiércoles contienen grandes cantidades de compuestos de fácil descomposición, cuya adición casi siempre resulta en un incremento de la actividad biológica" (pág. 3), estas características tienen que ver con aquellos microorganismos presentes en el suelo a sembrar como son hongos, bacterias, otros, cuya actividad biológica repercute en el mejoramiento de la estructura del suelo. 


\subsection{Sustentabilidad}

Una vez comprendidos los aportes de los abonos orgánicos a los suelos, cultivos y el ambiente es supremamente necesario entender e interiorizar en el desarrollo sustentable y así Calvente (2007) sostiene que "sustentabilidad es la habilidad de lograr la prosperidad económica sostenida en el tiempo protegiendo en la misma medida los sistemas naturales del planeta y proveyendo una alta calidad de vida para las personas" (pág. 4) se puede observar entonces tres dimensiones fundamentales que son: la economía, la sociedad y el ambiente, en tal sentido, según el planteamiento del autor debe haber una gran interrelación entre las mismas para lograr la sustentabilidad, por tanto al usar los abonos orgánicos como alternativa natural al uso de los químicos se afianza esta proposición.

En el mismo orden de ideas Calva (2007) plantea:

La sustentabilidad está asociada al principio de aprovechamiento de las propiedades del capital natural sin rebasar la capacidad del sistema para equilibrarse y garantizar la reproducción de las propiedades de los bienes naturales en periodos de tiempo posterior. Al asumirse que los recursos naturales tienen un impacto directo, tanto desde las perspectivas de la producción, el consumo y otros servicios intangibles como los estéticos; en el bienestar de los individuos, entonces el principio de la sustentabilidad pasa de ser una referencia física para adquirir una dimensión ética, política, económica y social (pág. 60).

Por lo tanto, no se debe tomar la sustentabilidad a la ligera, pues es ella la que, mediante primeramente el reconocimiento de una crisis ambiental y segundo la planificación, se puede lograr un desarrollo progresivo y sostenido sin afectar el ambiente.

Ahora bien, con mucho énfasis se explicó la alta conexión que hay entre la contaminación y los abonos químicos, desde contaminaciones comunes de 
agua, suelo y aire hasta intoxicaciones que pueden llegar a ser mortales para los seres humanos, entiéndase pues que la agricultura química no ayuda para nada al desarrollo sustentable, por el contrario, es un agravante de los problemas ya existentes.

En contraposición se propone la utilización de abonos orgánicos para lograr un desarrollo verdaderamente sustentable, esta proposición se fundamenta en todo este contexto teórico al entender las bondades naturales que aportan los mismos al ambiente muy inherente a la sustentabilidad.

\section{Metodología}

\subsection{Naturaleza de la Investigación}

La investigación está enfocada dentro del paradigma cualitativo debido a que a la misma le interesan los datos por sus cualidades, por lo que ocurre en cada uno de ellos y no tanto por sus cantidades. Está fundamentada en una visión holística de las realidades, lo cual implica comprender una realidad desde la relación que hay en cada uno de sus elementos y no desde la visión aislada de ellos.

\subsection{Método de la Investigación}

Lo anteriormente mencionado de una forma resumida, no es más que un fenómeno que ha venido, está y seguirá ocurriendo cada vez con más fuerzas, además hay otros puntos que intervienen en esta investigación como el uso indiscriminado de abonos químicos, la ingesta de hortalizas y frutos posiblemente contaminados, contaminación en general, entre otros, por lo que se intenta comprender y estudiar el hombre y su medio, lo que conlleva a trabajar con el método de investigación fenomenológico, en coherencia con lo planteado es preponderante citar al padre la fenomenología trascendental Husserl (1984) el cual planteó "la fenomenología surgió como una necesidad de explicar la naturaleza de las cosas" (pág. 28). Entonces no hay un mejor 
método de investigación que le fenomenológico para explicar a profundidad el tema desde la naturaleza del mismo.

Aun cuando el señor Edmund Husserl es el padre de la fenomenología como la conocemos, no está de más citar un autor que se halla expresado más recientemente, en tal sentido se tiene a Trejo (2012) el cual plantea: "La fenomenología lejos de ser un método de estudio, es una filosofía para entender el verdadero sentido de los fenómenos, pero con una secuencia de ideas y pasos que le dan rigurosidad científica" (pág. 101) y aunque el autor parece dilucidar que la fenomenología no es método de estudio, afianza que le da gran sentido a los fenómenos con rigor científico, aspecto que se necesita de forma imperante para abordar la situación de la alta utilización de los abonos químicos y el daño que ocasionan al ambiente y a los suelos.

\subsection{Unidades de Estudio}

Están referidas al contexto, característica o variable que se desea investigar. Es así como la unidad puede estar dada por una persona, un grupo $\mathrm{u}$ otro que contengan claramente los eventos a investigar.

Las unidades de estudio de esta investigación se definieron de tal modo que a través de ellas se pueda dar una respuesta completa y no parcial a la interrogante de la investigación por lo que son empleados actuales de la Escuela Técnica Agronómica Salesiana, poseen considerable tiempo laborando en la institución, tienen conocimientos técnicos sobre el tema, están directamente involucrados con el uso de abonos orgánicos o químicos y así se escogieron cuatro (4) profesores.

\subsection{Técnicas de Recolección de la Información}

Del mismo modo, al usar el método de investigación fenomenológico se concibe que se fundamenta en el estudio del mundo del ser humano, y debido a que es el hombre el que administra el uso de los abonos orgánicos o 
químicos, se utilizaron las siguientes técnicas de recolección de datos: la entrevista y la observación directa, por tanto según Vargas (2012), "La entrevista cualitativa permite la recopilación de información detallada en vista de que la persona que informa comparte oralmente con el investigador aquello concerniente a un tema específico o evento acaecido en su vida" (pág. 123), siendo así, la entrevista posibilita obtener de las unidades de estudio la información necesaria e importante para la investigación.

Con respecto a la observación directa, esta se utiliza en esta investigación debido a que, primero debe recordarse que la misma es un elemento fundamental de todo proceso investigativo debido a que en ella se apoya el investigador para obtener gran cantidad de información relevante y segundo es una técnica que consiste en observar atentamente el fenómeno, hecho o caso, tomar información y registrarla para su posterior análisis.

\section{Resultados y Discusión}

Al finalizar el proceso de análisis de las entrevistas, se debe considerar la interpretación de los significados de los relatos desde un enfoque social, ambiental y económico considerando las categorías: abonos orgánicos, abonos químicos y desarrollo sustentable abordados respectivamente.

Los abonos orgánicos en la Escuela Agronómica Salesiana son residuos de origen natural (animal o vegetal) que una vez incorporados al suelo son capaces de brindar a las plantas todos aquellos macro y microelementos necesarios para su desarrollo efectivo durante todo su ciclo productivo además de esto tienen la capacidad de modificar positivamente las características físicas, químicas y biológicas de los suelos mantenido así todas sus propiedades naturales, además de esto son supremamente fáciles y económicos de producir pues hasta las hojas secas pueden convertirse en una gran fuente de abono orgánico, y para terminar, el uso de abonos orgánicos debido a lo antes mencionado es inherente a la descontaminación y por ende 
también a la sustentabilidad, pues son naturales, económicos y descontaminan.

Los informantes son perfectamente claros cuando mencionan sin cesar que la institución misma cuenta con una gran cantidad de áreas altamente productivas de abonos orgánicos de excelente calidad como lo son: porcinocultura, ovinos y caprinos, pollos de engorde, lechería y bovinos de carne que producen diversos tipos de estiércoles y también hay áreas como jardinería que además de generar ramas y hojas secas producto de las constantes podas, se encarga fundamentalmente de la parte de composteros que consiste básicamente en recibir todos estos abonos antes mencionados, apilarlos ordenadamente por capas técnicamente planificadas, permitir su descomposición por algunos meses y tener como resultado uno de los mejores abonos que se pueden conocer para los suelos y las plantas el cual es el compost, que posteriormente está dispuesto a ser utilizado por las demás áreas que manejan cultivos.

En lo que respecta al desarrollo sustentable prepondera en los informantes la realidad muy marcada en los productores foráneos y en la Institución que hay una preferencia por el uso de los abonos químicos, pues es más fácil y rápida su utilización, pero también mencionan tajantemente que no son una alternativa viable ecológicamente y son incapaces de hacer mantener al suelo sus capacidades intrínsecas de fertilidad y que no se puede dejar de mencionar además que son una gran fuente de contaminación para el ambiente al ser un aditivo extraño o sintético que se le agrega a los suelos.

Asimismo, hablan con frecuencia entre los relatos que al usar los abonos orgánicos si podría lograrse un desarrollo verdadero interno o endógeno de la institución pues todo se produce y maneja dentro de la misma. Por plantearlo de esta forma, existen áreas productoras de estiércoles y restos vegetales, luego pasan a otra área que los procesa y transforma en compost y por último pueden ser usados en aquellas áreas que usan fertilizantes 
químicos, como las áreas de horticultura y fruticultura, incluso en el área de cultivos extensivos (Maíz), disminuyendo así el uso de los mismos y al dejar de usarlos se lograría lo que plantea la investigación y es el desarrollo sustentable de la Institución ya que se lograría un equilibrio pleno entre las tres dimensiones de la misma que son lo social, económico y ambiental.

En lo social la institución podría enseñar a sus estudiantes las bondades de los abonos orgánicos, en lo económico al prescindir de los fertilizantes químicos la escuela lograría ahorrarse una cantidad considerable de dinero y en lo ambiental se lograría frenar el deterioro progresivo de los suelos haciéndolos más fértiles para que las futuras generaciones puedan aprovechar ese suelo y además no habría contaminación por efecto de envases de fertilizantes ni habrían derrames accidentales de químicos a abrevaderos de animales productivos, observando este hecho muy proclamado por los informantes, se puede deducir que se estarían dando pasos agigantados hacia un desarrollo sustentable de la Escuela Agronómica Salesiana.

Lo que tiene que ver con los abonos químicos en la Escuela Agronómica Salesiana son productos sintéticos o artificiales adquiridos por la misma a elevados precios económicos, que son elaborados a partir de sustancias no orgánicas y una vez aplicados al suelo le aportan a las plantas algunos nutrientes para su desarrollo, según los informantes son varias las áreas donde se suministran los mismos, desde cultivos extensivos hasta los frutales y las hortalizas, estos dos últimos son hacia donde se centra la investigación.

Impera en los entrevistados el hecho indiscutible de que con el uso de fertilizantes químicos eventualmente se degradan y empobrecen los suelos, no permitiendo así el desarrollo de los futuros cultivos a largo plazo, además mencionan los impactos ambientales que causan los mismos, desde contaminaciones por dejar los envases en los alrededores hasta el problema grave donde se vierten accidentalmente fertilizantes químicos como la Urea (Nitrógeno al $46 \%$ ) a lagunas donde circundan animales de ordeño, que al 
consumir dicha agua no solo podría intoxicarlos sino que más importante podría causarles la muerte casi instantánea según los informantes

Por lo tanto pues, se consideran altamente perjudiciales los abonos o fertilizantes químicos, ya que son un menoscabo económico y de salud para la institución, no obstante redunda entre los relatos de los informantes las bondades de los abonos orgánicos, entre los cuales resaltan el mantenimiento y el mejoramiento de los suelos, desarrollo muy aceptable de los cultivos tratados y el ahorro de la institución al prescindir de los abonos químicos y comenzar a profundizar el uso de abonos orgánicos.

\subsection{Consideraciones finales}

De acuerdo con la aplicación de las entrevistas a los sujetos claves, de la observación directa en los predios de la Escuela Técnica agronómica Salesiana de Barinas, de la investigación documental previa, y del tratamiento científico, social, económico y ambiental a la información y datos recabados, se evidenciaron las siguientes conclusiones:

- Los abonos químicos se suministran en grandes cantidades en la institución sobre todo en los cultivos como el maíz, frutales y hortalizas $y$ aun cuando estos ayudan en el desarrollo de los cultivos son responsables directos del deterioro progresivo de los suelos fértiles del plantel.

- Los abonos orgánicos son indispensables para la conservación de los suelos e inherentes al cuidado del medio ambiente, aunque más lento, permiten un desarrollo satisfactorio y ecológico de los cultivos, son producidos a gran escala en la Escuela por sus diversas áreas pecuarias, así como las áreas de producción vegetal. Los encuestados son tajantes en el hecho de que si se aumenta el uso de los mismos y de disminuye el uso de los químicos se lograría frenar el deterioro de 
los suelos además ocurriría un desahogo en la economía de la institución.

- La sustentabilidad viene precedida de un equilibrio e interacción entre lo social, económico y el ambiente, al mantener el uso de los abonos químicos se mantiene un desequilibrio inminente pues ni son económicos, ni cuidan el ambiente y por tanto no ayudan en lo social, en resumen, no se lograría el propósito del Informe de la Comisión Mundial sobre el Medio Ambiente y el Desarrollo "Nuestro Futuro Común" presidido por Brundtland, G. (1987) el cual plantea que el desarrollo sustentable o desarrollo duradero como también lo llama dicha comisión "es aquel que satisface las necesidades de la generación presente sin comprometer la capacidad de las generaciones futuras para satisfacer sus propias necesidades" (pág. 59), siendo así, con los abonos químicos se satisface muy bien las necesidades actuales, pero producto del deterioro y contaminación que causan no se asegura que las generaciones futuras satisfagas las suyas.

A partir de las conclusiones y de los resultados obtenidos de esta investigación tan interesante se desprenden importantes recomendaciones dirigidas fundamentalmente a la Escuela Agronómica Salesiana "San José" como lo son:

- Seguir manteniendo y mejorar el área de composteros presente en la escuela, la cual ya se ha explicado que está dispuesta para la recepción de material orgánico como estiércoles y restos de animales y vegetales y con ellos producir abonos orgánicos de excelente calidad para los cultivos frutales y hortícolas. 
- Elaborar un plan para reducir exponencialmente el suministro de fertilizantes químicos a los suelos y aumentar el uso de abonos orgánicos que están muy presentes en la misma institución.

- Para culminar, pero no menos importante, la agricultura, si se quiere, puede considerarse la madre del ser humano o su pilar fundamental para existir, debido a que ya se sabe que la base de la subsistencia de las personas es el agua y la comida, la ingesta diaria de alimentos, estos que con todas su características intrínsecas por excelencia pueden producirse sola y únicamente mediante la agricultura, en suelos, con manejos agrícolas efectivos, las plantas, frutos y demás vegetales que se consumen vienen de los suelos, las carnes se originan del ganado vacuno el cual se alimenta fundamentalmente de pasto y este por su puesto solo se produce en el suelo, el mismo suelo que los humanos usan para la actividad agrícola a diario, el mismo suelo que cada vez se degrada y contamina más a causa de la frecuente fertilización con químicos, pero que indudablemente se tiene a la mano una opción natural, económica y ecológica como lo son los abonos orgánicos, se exhorta pues a la institución en estudio y al amigo lector que guste leer esta investigación a poner ese granito de arena y abonar sus cultivos con la misma materia orgánica que produzca su casa o finca.

Se considera pues impostergable el hecho de comenzar a usar intensivamente los abonos orgánicos y dejar atrás los químicos que tanto daño hacen a la Institución y a la humanidad.

\section{Referencias}

Brundtland, G. (Coord). (1987). Informe de la Comisión Mundial sobre el Medio Ambiente y el Desarrollo "Nuestro Futuro Común". Nairobi: 
Organización de Naciones Unidas.

Calva. L. (2007). Sustentabilidad y Desarrollo Ambiental. Agenda Para el Desarrollo, Universidad Nacional Autónoma de México. México.

Calvente, A. (2007). El concepto moderno de sustentabilidad. [Documento en línea]. Recuperado de:

http://www.sustentabilidad.uai.edu.ar/pdf/sde/uais-sds-100-002\%20\%20sustentabilidad.pdf

Duran, M. (2015). Tipos de abonos orgánicos. Recuperado de: http://www.ecoagricultor.com/tipos-de-abonos-organicos/

Gonzales, F. (2011). Medio ambiente y desarrollo sostenible. [Documento en línea]. Recuperado de:

http://fgonzalesh.blogspot.com/2011/01/contaminacion-porfertilizantes-un.html

Husserl, E. (1984). Crisis de las ciencias europeas y la fenomenología trascendental. (El manuscrito data de 1935-1936, publicado originalmente en alemán en 1962). México: Ediciones Folios. Recuperado de: http://www.redalyc.org/pdf/340/34024824004.pdf

Martínez, C., y otros (1999). Lumbricultura y abonos orgánicos. México: Colegio de postgraduados de la Universidad Autónoma Chapingo.

Torres, C. y otros (2002). Manual Agropecuario, Tecnologías Orgánicas de la Granja Integral Autosuficiente. Editorial Comarpe Internacional CA. Trejo, F. (2012). Fenomenología como método de investigación. Vol. 11, No. 2, 98-101. México.

Trinidad, A. (2011). Abonos orgánicos. Secretaría de Agricultura, Ganadería, Desarrollo Rural, Pesca y Alimentación. México.

Vargas, J. (2012). La Entrevista en la Investigación Cualitativa: Nuevas Tendencias y Retos. Volumen 3, Número 1. Centro de Investigación y Docencia en Educación Universidad Nacional, Costa Rica. 


\section{Ángel Asdrúbal Torres Moreno \\ e-mail: angelasdrubal 44@hotmail.com}

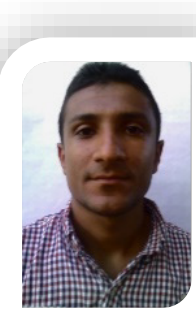

Nacido en Palmarito estado Apure, Venezuela. cursa estudios de Maestría en Educación, Ambiente y Desarrollo en la Universidad Pedagógica Experimental Libertador, núcleo Barinas, Título de Profesor en Educación Agropecuaria en la misma universidad (2013), Técnico Medio en Cencías Agrícolas, Escuela Técnica Agronómica Salesiana "San José" Barinas (2006).

Actualmente se desempeña como profesor de campo en la Escuela Técnica Agronómica Salesiana "San José" Barinas, en la asignatura de Frutales, Raíces y Tubérculos, desde el 2007.

El contenido de este manuscrito se difunde bajo una Licencia de Creative Commons ReconocimientoNoComercial-Compartirlgual 4.0 Internacional 